Conclusions Demand for inpatient care in CF is hugely variable, with a high burden of care being concentrated in a small proportion of patients. CF diabetes and low lung function are risk factors. These patients have high mortality. Kaftrio is likely to decrease the need for inpatient care for many of these, but a small cohort with unresponsive genes will continue to require high levels of specialist inpatient care.

\section{P115 THE DIAGNOSIS AND MONITORING OF CYSTIC FIBROSIS LIVER DISEASE IN A WEST OF SCOTLAND CF COHORT}

P Holland, DJ Leith, M Priest, S Thomson, S Bicknell, G MacGregor. Queen Elizabeth University Hospital, Glasgow, UK

\subsection{6/thorax-2021-BTSabstracts.224}

Introduction Cystic fibrosis [CF] is a systemic disease resulting from loss of function of the CFTR protein. Despite being a significant cause of mortality, CF liver disease [CFLD] remains under recognised.

This study determined the prevalence of CFLD and CFLDcirrhosis in our cohort of adult CF patients and the adherence to local surveillance protocols for diagnosing and monitoring these conditions.

Methods The study identified 270 patients with a diagnosis of CF in the West of Scotland Adult CF Service. Electronic clinical records were reviewed to determine the prevalence of pre-existing diagnoses of CFLD and assess for evidence of cirrhosis. Appropriate liver monitoring and gastroenterology service involvement, as per locally agreed protocols, was reviewed.

Results The mean age of the cohort was 32 years (range 16$71)$ and $145(54 \%)$ were male. A pre-existing diagnosis of CFLD occurred in 80/270 (30\%) patients, 23 (29\%) of whom had evidence of CFLD-cirrhosis - $9 \%$ of the total cohort.

The local standard of yearly and 6 monthly liver function tests [LFT] in non-CFLD and CFLD/CFLD-cirrhosis patients respectively was met in 241/270 individuals (89\%).

The local standard of 5 yearly, 2 yearly and 6 monthly ultrasounds in non-CFLD, CFLD and CFLD-cirrhosis patients respectively was achieved in 190/270 individuals (70\%).

Of those with CFLD/CFLD-cirrhosis, regular follow up with or previous discharge from gastroenterology services occurred in $34 / 80$ patients (43\%).

\begin{tabular}{llll} 
Abstract P115 Table 1 & & & \\
\hline & $\begin{array}{l}\text { Non- } \\
\text { CFLD }\end{array}$ & $\begin{array}{l}\text { Non- } \\
\text { Cirrhotic }\end{array}$ & $\begin{array}{l}\text { CFLD } \\
\text { Cirrhosis }\end{array}$ \\
& {$[190 /$} & $\begin{array}{l}\text { CFLD } \\
{[23 / 270 ;}\end{array}$ & $\begin{array}{l} \\
{[57 / 270 ;}\end{array}$ \\
& $270 ;$ & $9 \%]$ \\
& $70 \%]$ & $21 \%]$ & \\
\hline LFT Standard Met & $173 / 190$ & $49 / 57$ & $19 / 23$ \\
[Yearly LFT Non-CFLD; & $(91 \%)$ & $(86 \%)$ & $(83 \%)$ \\
6 monthly LFT CFLD/CFLD-cirrhosis] & & & \\
Ultrasound Standard Met & $138 / 190$ & $35 / 57$ & $17 / 23$ \\
[5-yearly US Non-CFLD; 2-yearly US Non-cirrhotic & $(73 \%)$ & $(61 \%)$ & $(74 \%)$ \\
CFLD; 6-monthly US CFLD-cirrhosis & & & \\
Gastroenterology Referral & N/A & $22 / 57$ & $12 / 23$ \\
& & $(39 \%)$ & $(52 \%)$ \\
\hline
\end{tabular}

Conclusions CFLD is a source of significant morbidity and mortality in CF patients and affects $30 \%$ of our patient cohort, with evidence of cirrhosis in 29\% of CFLD patients. However, a risk of delayed or missed CFLD diagnosis has been identified for patients not receiving 5 yearly screening ultrasound and regular LFT monitoring. Additionally, patients with CFLD or CFLD-cirrhosis are not always receiving more intensive liver monitoring such as increased ultrasound and LFT testing frequency. Improvements also need to be made to ensure all CFLD/CFLD-cirrhosis patients are referred to gastroenterology services.

We will strengthen existing protocols guiding liver monitoring and referral pathways for non-CFLD, CFLD and CFLDcirrhosis patients.

\section{P116 A NOVEL TOOL TO INTERPRET THE INCREMENTAL SHUTTLE WALK TEST (ISWT) IN A CYSTIC FIBROSIS (CF) PAEDIATRIC POPULATION}

1J Simpson, ${ }^{2} \mathrm{~F}$ Robinson, ${ }^{2} \mathrm{~A}$ Burrill, ${ }^{2} \mathrm{~B}$ Heyeer, ${ }^{1} \mathrm{~A}$ Toffin, ${ }^{2} \mathrm{P}$ Kenia, ${ }^{2} \mathrm{M}$ Desai, ${ }^{2} \mathrm{P}$ Nagakumar. 'Physiotherapy Department Birmingham Women's Hospital, Birmingham, UK: ${ }^{2}$ Respiratory Department Birmingham Women's Hospital, Birmingham, UK

\subsection{6/thorax-2021-BTSabstracts.225}

Introduction Cardiopulmonary exercise testing (CPET) is the gold standard for the assessment of exercise tolerance but is not widely available. There is increased recognition of the prognostic value of exercise tolerance in patients with $\mathrm{CF}$ but there is a lack of normative values for other exercise tests within the literature. The lack of such data prompted the development of the Birmingham ISWT tool to provide more insight into ISWT results gained and identify those that would benefit from an exercise intervention. We developed the tool incorporating published equations that predict $\mathrm{VO}_{2}$ peak for distance achieved in an ISWT (Fernanda-PaedPul-2019) and compared this to published normal values for $\mathrm{VO}_{2}$ peak by age and sex (Ten-Harkel-EurJCardiovasc-2011). The tool provides thresholds that enable a patient's results to be rated as green (predicted $Z$ score $\geq-1$ ), amber (predicted $Z$ score -1 to -2 ) or red (predicted $Z$ score $<-2$ ) based on their ISWT distance.

Methods The tool was used to evaluate the most recent ISWTs completed for all CF patients attending our centre aged 8years and over $(n=112)$.

Results 112 ISWTs were completed in our patient group. 65 of the 112 of the tests completed were deemed either maximal $(n=52)$ or non-maximal but the patient completed all 15 levels $(n=13)$ and could be evaluated using the tool, with 49 rated as green, 12 amber and 4 red. In those rated amber or red, 9 stopped due to leg fatigue, 3 due to difficulty in breathing and 4 for either safety or unknown factors. The remaining 47 results were assessed as non-maximal and therefore not evaluated with 35 stopping for safety issues, 4 technical issues, 3 because of foot $/$ nnee/back pain and 5 for unknown factors.

Conclusions We have shown that the Birmingham ISWT tool proved easy and quick to use and enabled us to highlight those that would benefit from intervention. $75 \%$ of our cohort had good levels of fitness. Leg fatigue was the most common reason for stopping the test rather than shortness of breath. Future plans include validating the tool and developing targeted interventions to improve physical fitness in children with CF. 